
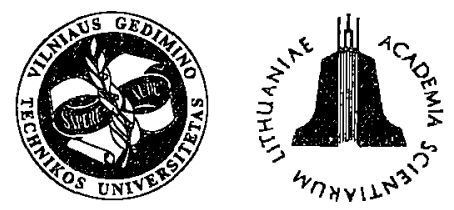

ISSN 1648-4142 TRANSPORT

http:/www.vtu.ti/english/editions

TRANSPORT - 2002, Vol XVII, No 6, 205-218

\title{
MODELS OF FREIGHT TRANSPORT SYSTEM DEVELOPMENT
}

\author{
Adolfas Baublys ${ }^{1}$, Algimantas Smičius ${ }^{2}$ \\ Transport Research Institute, Vilnius Gediminas Technical University, \\ Plytines g. 27, LT-2040 Vilnius, Lithuania.
}

Received 200205 25; accepted 20021021

\begin{abstract}
The article analyses the following issues: 1) Basic factors determining transport system development; 2) Transport system services and their assessment; 3) Goods handling transport system as a research object. The objective of goods handling transport system development and improvement is to establish a general goods handling transport system of the country ensuring satisfaction of national needs for high quality transport service in this field; 4) Models of transport modes interaction; 5) Determinate models for main transport network development; 6) Model of development of private freight road transport.
\end{abstract}

Keywords: freight transport, development of transport system and network.

\section{Introduction}

Lithuanian transport sector undergoes an essential reform and restructuring endeavouring the integration of the national economics into the processes of European and global labour division at market - based transformation of the Lithuanian economy.

The main objective of the formulated national transport system development programme is the creation of a solid multimodal transport system and its integration into the West - European market of transport services as well as the promotion of transit freight flows from abroad through the territory of Lithuania.

Economic situation in Lithuania requires rapid development of transport system because the essential changes have taken place in the territorial distribution of transport flows (flows shifted from the East to the West) as well as in the structure of inner flows during the recent ten years.

Until now the Lithuanian transport sector was rather isolated from the transport system of the countries of Western Europe. There is a lack of modern transport infrastructure, transport means adequate to the requirements of international technical, technological and ecological standards, also there is not a sufficient number of specialists able of efficient international transport operation. The integration of Lithuanian transport into the European transport system has to be inseparable from the formation of integrated international transport corridors of the entire Baltic Sea region countries relating with the transport infrastructure objects of the Western and Northern Europe.

${ }^{1}$ E-mail: abaublys@takas.1t

${ }^{2}$ E-mail: center@aaa.1t
On the other hand the reconstruction, restructuring and development of the transport sector is a constituent part of the process of entire Lithuanian economy and its integration into the European Union.

The geopolitical situation of Lithuania gives a good opportunity to play an important role in transit transport in the North - South and East - West directions. Situated on the coast of the Baltic Sea, located in the middle of the continent Lithuania is on the crossroad between the developed countries of the Western Europe and the countries of the Eastern Europe.

For freight flows service of the East - West direction Lithuania has the Klaipeda port - one of the few ice-free ports on the North - Eastern coast of the Baltic Sea. The Klaipeda seaport has one more advantage (if compared with the neighbouring ports) - it has a good link with the national highways and railways adequate to international standards. Also Lithuania functions as a transit country for the transport to/from the Russian Kaliningrad port.

Transport, as well as its development programme, are very dynamic and changeable objects, therefore scientifically grounded methodological fundamentals have to be created enabling efficient co-ordination of strategic as well as technical and technological principles of programme implementation.

Therefore, the aim of our research is the adaptation of systematic analysis methods making them relevant for the periodical correction and concretising of the transport sector development strategy of the Republic of Lithuania.

Our research aims at the adaptation of systemic analysis and modelling principles of Lithuanian transport system development in the conditions of transition to market economy bearing in mind the fact that Lithuania is a tran- 
sit country and it is creating a principally new functioning transport system consisting of the road, railway, inland waterways, maritime and air transport.

It is the first time that systemic analysis methods and models based on them are adapted with certain corrections (for the adaptation to the transition to the market conditions) for the whole Lithuanian transport sector as for a complex system (not for its separate parts). With the help of the methods and models Lithuanian transport system development implementation strategy was modelled and separate components were elaborated.

After the review of the reference list it is noted that there are only those models which are used for transport planning and development at the conditions of stable market $[1-4]$.

\section{The Main Factors Determining the Transport Sys- tem Development}

Transport research may be efficient and practically useful if based on the factors preconditioning the development of transport system, as well as on the awareness of its nature and knowledge of its analytical (methodological) instruments which are worked out by transport engineering, transport economics, management, mathematics, statistics, etc. It is very important to make use of the knowledge (at axiom level) accumulated by different trends of science. To such (i.e. those preconditioning the development of transport system) we attributed the following statements [1]:

1. A great number of interactive elements are functioning in transport system, which causes the occurrence of a great stochastic effect and an opportunity to pursue goals by different means.

2. In the course of transport system it is necessary to introduce a dynamic element or time bound connections, whereas the very fact of iransport system undergoing changes is related with the delayed reaction, feedback and temporary disbalance.

3. Transport demand is always derived from the demand for stock availability at sites for production process, availability of goods at their consuming places or, as it is in the case of passenger transport, possibility to reach the place of destination. Therefore the economical analysis of demand derivative is an important factor of the demand assessment and analysis of intermodal competition.

4. Transport services (product) can not be collected and stored, the processes of their production and application are not separated technologically.

5. A significant share of advantage (efficiency) of transport system does not reflect in statistical indices, but manifests itself through the characteristics of economic sectors served by transport.

6. The adaptation of a transport system to the changes of the social-economical environment most often proceeds with certain lateness. Real assessment of the lateness (inertia) is necessary for working out of transport policy recommendations, improvement of transport technologies, programming and definition of transformation priorities, necessary costs, as well as for the working out of concrete investment projects.

7. Functioning of transport objects is characterised by inertness: for some time they may operate on inertia, on the account of overloading and production quality, thus retaining the image of demand satisfaction.

8. Transport is a very capital consuming sector of national economy. Long terms of designing, creation and functioning are characteristic to the objects of transport infrastructure.

9. As a rule capacities of transport infrastructure objects are increased by stages. Having covered one stage (for instance, having constructed a new road) a reserve of transportation capacities is made, which is assimilated not at once, but gradually, during several years.

10. Transport realises the main share of external economic relations, plays an active role in the process of international labour division.

The enumerated peculiarities determining transport policy are characteristic to the entire transport system. It is evident that every transport mode has its own peculiarities.

\section{Transport System Services and Their Assessment}

The development of a transport system and its services are determined by the distribution of social and economic activities in space, i.e. the distribution and characteristics of inhabitants, industries, consuming and distribution centres.

For the emphyric research of transport system services it is important to define properly the demand and supply which would enable to assess these categories unambiguously. The following definitions of these categories are possible.

The demand of transport is defined by: a) initial and final point of movement; b) number of units and weight of goods to be transported; c) transportation time; d) specific characteristics of services (according to the client's request).

Transport supply is defined by: a) initial and final point of movement and selection of route; b) offered capacities for goods transportation and their handling; c)transportation time; d) characteristics of service supplied.

These definitions of categories of transport services' demand and supply enable to apply the unified indices of their assessment.

For general characteristics of transport activities natural and monetary indices are used general to all transport modes and specific indices of each mode $[5,6]$. 
The most important general natural indices are: transportation volume $Q_{r}$ (measured by tons for freight transport) and the turnover of freight $P_{r}$ (measured by tonkilometres), here $r$ - transport mode.

For each freight type $k$ transportation volume by transport mode $r$ will be

$$
Q_{r}^{k}=\sum_{i, j} Q_{i j}^{k r}
$$

here transportation volume of freight $k$ type by the transport mode $r$ from the point $i$ to the point $j$ $(k=1,2, \ldots, z ; i=1,2, \ldots, m ; j=1,2, \ldots, n)$.

The circulation of $k$ type freight for the transport mode $r$ equals:

$$
P_{r}^{k}=\sum_{i, j} P_{i j}^{k r},
$$

here $P_{i j}^{k r}-k$ type freight circulation in its transportation from point $i$ to point $j$ by the transport mode $r$.

The freight circulation $P_{i j}^{k r}$ and transportation volume $Q_{i j}^{k r}$ interrelate as follows:

$$
P_{i j}^{k r}=Q_{i j}^{k r} l_{i j}^{k r}
$$

here $l_{i j}^{k r}-k$ type freight transportation distance from the point $i$ to the point $j$ by the transport mode $r$. Then the general transportation volume $Q_{r}$ (according to the freight type) is the general freight circulation $P_{r}$ by the transport mode $r$ obtained as follows:

$$
\begin{aligned}
& Q_{r}=\sum_{k} Q_{r}^{k}=\sum_{i, j, k} Q_{i j}^{k r}, \\
& P_{r}=\sum_{k} P_{r}^{k}=\sum_{i, j, k} P_{i j}^{k r}=\sum_{i, j, k} Q_{i j}^{k r} l_{i j}^{k r} .
\end{aligned}
$$

For each transport mode $r$ an important characteristics is a medium $k$ type freight transportation distance:

$$
l_{v i d .}^{k r}=\frac{P_{r}^{k}}{Q_{r}^{k}}=\frac{\sum_{i, j} Q_{i j}^{k r} l_{i j}^{k r}}{\sum_{i, j} Q_{i j}^{k r}}
$$

and medium distance of transportation of all the freight types is:

$$
l_{v i d .}^{r}=\frac{P_{r}}{Q_{r}}=\frac{\sum_{i, j, k} Q_{i j}^{k r} l_{i j}^{k r}}{\sum_{i, j, k} Q_{i j}^{k r}} .
$$

Analysing transport system operation it is necessary to define the medium distance of transportation of each type freight not by a separate transport mode, but by that of the entire transport system:

$$
l_{\text {vid. }}=\frac{P^{k}}{Q_{\text {pirm. }}^{k}}
$$

here $P^{k}=\sum P_{r}^{k}$ - general $k$ type freight circulation in the transport system; $Q_{\text {pirm. }}^{k}$ - initial $k$ type freight transhipment in the transport system.

The initial transhipment may be equal or less according to the transportation volume $Q^{k}=\sum_{r} Q_{r}^{k}$, as in the latter one there may be repetitive transportation of the same freight (for instance, by passing from one transport mode to another).

Wishing to characterize the work of the whole transport system we have to apply the general indicative of freight circulation (that of all transport modes and of all freight types) $P=\sum_{k} P^{k}=\sum_{k, r} P_{r}^{k}$ and general transportation volume $Q=\sum_{r} Q^{r}$.

It is also possible to speak about the medium distance of all freight transportations. However such "mediumised" distance (especially of all the transport modes) has not much practical value except the modelling cases.

Applying the formula (1)-(8) it should be born in mind that amounts used in them by which calculations are exercised are related to several transport modes.

The volume of transportation consists of freight reception from freight consignors and from other transport modes according to the general transportation documentation or, on the consignee's authorisation, as well as basing on separate documents.

In its turn the reception from the freight consignee may be initial (of imported goods as well) and repetitive from the storage points. Accordingly the formulae (7) and (8) may determine two principally different medium transportation distance meanings: simple (technological) and economic. The simple medium distance is calculated by the formulae (6) and (7) for one transport mode and according to the distances from the freight consignor to the recipient. Medium economic transportation distance is defined according to the formula (8) taking into account the distance from the producer to the consumer and not according to the total consignment, but only according to the initial one.

Conditional natural indices of a transport system are defined by the amounts $R_{s}$, to which various concrete indices $R_{k}$ are adjusted using correction coefficients $\gamma_{s}(s=1,2, \ldots, S)$

$$
R_{s}=\sum_{s=1}^{s=S} \gamma_{s} R_{k}
$$


Adjusted tonnekilometers are attributed to the conditional natural indices. Usually it is [7]: 1 tonkilometer $=1$ adjusted tonkilometer; 1 passenger kilometre (in the railway, maritime and inland water transport) $=1$ adjusted tonkilometre; 1 passenger kilometre (in the road transport $)=0,25$ of adjusted tonkilometer, etc. For the indicated concrete indices: in the railway transport $R_{1}^{g}$ is measured by tonkilometres, $R_{2}^{g}$ - by passenger kilometres, $\gamma_{1}^{g}=\lambda_{2}^{g}=1$; in the road transport $R_{1}^{a}$ is measured by tonkilometres $\gamma_{1}^{a}=1, R_{2}^{k}$ - by passenger kilometres $\gamma_{2}^{k}=0,25$.

It should be noted that the problem of stability or differentiation of adjustment coefficients $\gamma_{s}$ in regard of various transport analysis and planning issues is not solved. Therefore, it would hardly be right if we select one and the same $\gamma_{s}$ meaning determining the volume of transport system's adjusted operation which will be used in the calculation of the medium cost price, work consumption, etc. Actually, for efficiency calculation, the proportion of 1 passenger kilometre and 1 tonkilomtre of freight transportation, for instance, in the maritime and inland waterways transport has to exceed tenfold than, for instance, calculating the cost, whereas in regard of live labour demand one passenger kilometre for these transport modes is once or twice more labour consuming.

Therefore, it is not accidental that for the definition of labour efficiency we engaged the adjustment coefficients $\gamma_{s}$, namely:

in railway transport $-\gamma_{1}^{g}=1, \gamma_{2}^{g}=2$;

in maritime transport $-\gamma_{1}^{m l}=1, \gamma_{2}^{m l}=50$;

in inland waterways transport $-\gamma_{1}^{u}=1, \gamma_{2}^{u}=10$;

in road transport $-\gamma_{1}^{k}=1, \gamma_{2}^{k}=0,4$.

The analysed system of natural and conditionally

natural indices may be applied for the definition of transport system functioning characteristics in different aspects.

It should be noted that practically and scientifically long and intense discussions are continuing as for which activity index should be considered the main one - that of transportation volume or freight circulation, i.e. tons or tonkilomtres. It is considered that from the practical point of view this problem is actually solved. In statistics the main natural indices are considered the following: transported freight volumes in tons and transported numbers of passengers. Freight circulation by tonkilometres according to the transport modes characterizes the operation of transport and is applied in calculation of material resources and in solution of other problems.

However, the discussions on this topic have not yet been finalised. At present the idea of measuring the volumes of transport production (services) by the following amount is raised:

$$
\sum P v^{a}
$$

here $P$ - freight circulation, tkm; $v$ - delivery speed; $a$ - constant parameter.

The research work [7] proposes $a=2$, also it is mentioned that other authors propose $a=1$. The logics of the reasoning is as follows: it is necessary to assess the difference of delivery speeds, as it is not the same how much time the transportation takes. To my opinion here different issues are again mixed: definition of transport production volume, assessment of efficiency and quality of transportation process.

Requirements for freight transport services are presented in Fig.

\section{Freight Transport System}

The aim of the development and perfection of the freight transport system is the creation of a general freight transport system meeting the demands of qualitative transport service.

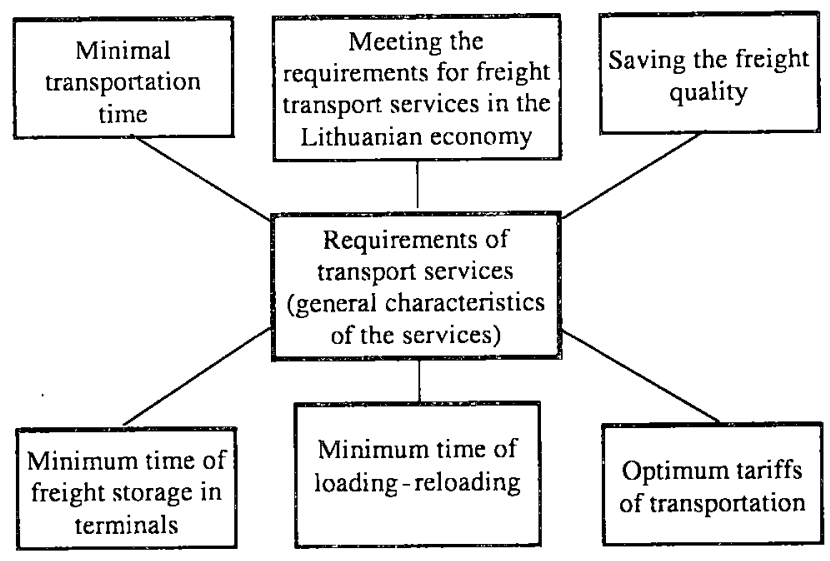

Requirements for transport services

One of the main trends in solving this problem is the planning and technological management of separate transport modes in their common interaction.

The analysis of freight transport system maintains that:

1. Transport system is a complex developing object which is subject only to the methods of systemic analysis.

2. Management of a system is the process of formation of efficient functioning (basing on efficiency criterion) of the system. The main objective of the management of transport system and freight flows is the formation of its characteristics guaranteeing the necessary quality of transport flows servicing at the least costs.

Since there is no universal criterion of functioning of transport system and freight flows, certain difficulties occur in the selection of the system parameters observed during the time of its functioning. As a rule the observed parameters should be the work of transport system elements deciding upon the functioning of transport system and the quality of handling freight flows. 
For the selection of a transport system management methods - more specifically - the rational management methods of national freight transport development use mathematical methods of the operation research.

Let us presume that for the national freight system research we have:

$$
U=\left\{U_{i}, i=1, N\right\}-\quad \text { the set of methods for solving }
$$

the problems of national freight system development;

$S=\left\{S_{i}, i=1, N\right\}-$ the set of expenditures corresponding to the set $U$;

$\delta=\left\{\delta_{i}, i=1, N\right\}-$ the set of expedience indices with the application of the methods of the set $U$ for the research and development of freight transport system;

$$
R=\left\{R_{j}, j=1, M\right\} \text {-maximum amounts of resources }
$$

available to the state for the freight transport system development.

The objective of the rational management of national transport system development would be formulated as follows: from the set $U=\left\{U_{i}, i=1, N\right\}$ to find a subset $\Delta u \in U$, characterized by minimisation of functional to the subset

$$
\begin{aligned}
& \Delta \sigma \in \delta \text { and } \Delta s \in S: \\
& C=f_{1}(\Delta U, \Delta \delta),
\end{aligned}
$$

considering the limitation:

$$
R \geq f_{2}(\Delta u, \Delta s) \text {. }
$$

Thus, selecting the management issues of the national freight transport development and their solution methods it is necessary to make a purpose function (11), to define the set of development management objectives and their solution set $U_{0}$, constantly analysing the national freight transport system functioning and freight flows.

From the point of view of the theory of systems the national freight transport system is a complex system with all its indices peculiar to such a system and this determines the application of research methods in the research of national freight system.

The structure of a national freight transport system consists of the set of system elements and connections between them. This system has the following main characteristics:

- Freight flows (bids for transportation, their aerial distribution in regard of time);

- Transport network and the fleet of units of different transport modes;

- Plans of the traffic of units of separate transport modes (their distribution in the transport network, traffic timetables).

In the process of research of the problems of transport system functioning, in the preparation of the development programme, in the decision-making the following scheme of a systematic analysis was applied:

1. The clarification of the problem, its formulation and structuring.
2. The research of specifics of the object, investigation of its inner and outer connections in aspects of time, space, structure, etc.

3. The formulation of problem solving issues, the determination of criteria and their hierarchic connections, the ability to group and assess them qualitatively,

4. The definition of alternative ways of reaching the aims and determination of the main limitations.

5. The collection of initial information, the assessment of reliance and particularity, the ability to expand information and enhance preciseness.

6. The elaboration of models of different types, the quantitative analysis of the main structural elements, envisaging of costs and results related with altematives.

7. The calculations according to the models, the synthesis of quantitative and qualitative analysis, the corrections exercised by experts and decision-making, if needed - correction of models, of initial information, reiteration of calculations and synthesis of results.

In the first stage according to the structuring feature usually there are problems of four kinds: standard, well structured, poorly structured and non-structured. Accordingly the inventory problem solving varies.

It should be noticed that all the mentioned stages are not necessary apriori, but their necessity is determined during the course of system analysis.

Using the principles of system analysis for the research of a transport system it is necessary to observe the following:

1. The assessment of transport system complexity peculiarities. Transport system in general and its sub-systems consist of a great number of interrelated elements and the number of types of these elements is also sufficiently large. The interaction of separate transport modes - irrespectively of their own interests and aims - actually determines the occurrence of systemic effects. (Systemic effect will be defined as that which is not to be singled out from the features of separate transport modes, but defined only in their interface.)

In the analysis of a complex transport system it is very important to assess the systemic effects because the orientation towards the local advantage of separate transport modes leads to the decision-making disadvantageous to the whole system in general. Therefore, a certain mechanism for co-ordination of interests of all transport users is necessary. Adjustment of the parameters of this mechanism should guarantee the solutions expediency for all actors of a transport system. Given such effects it leads to the situation that the effect of the whole system is not in congruity with the sum of its separate effects. In this case the so-called effect of complex systems integrity manifests itself. The concentration and specialisation of transport process may serve as an example of such effect.

2. The assessment of hierarchic structure of a national transport system. Aspects of systemic compatibility and structural versatility of hierarchic structure features should be born in mind. The requirements of hierar- 
chic compatibility do not mean an equivalent congruity of transport system indices at various hierarchic levels (those of time, space, etc.). On the contrary, it is considered that at various hierarchic levels the degree of aggregation of the main characteristics of the system would be different, i.e. it concerns those indices which are used for the planning and development of a transport system, projecting and assessment of efficiency of transport system activities. Separate characteristics (indices) may be congruous at different hierarchic levels. For instance, used at all levels indices, such as those of freight circulation, freight volumes, etc.

3. The assessment of peculiarities, formalisation possibilities and manageability of transport system elements at all hierarchic levels. It is very important to assess the fact that the man is involved in a transport system. The man acts as a service supplier and consumer on one hand and as a management subject on the other hand, therefore not all the processes of a transport system may be formalised.

4. The analysis of the consequences of solutions. Consequences of solutions should be analysed not only within the limits of the technological mechanism of interacting transport modes, but also in transport users', ecological, political and other fields as well. It is important to control whether the solutions made in a transport system do not cause other economic functioning indices to trespass the limits of permissible state.

5. Transport system may be investigated in two aspects: 1) as a relatively separate dynamic complex system developing according to the features peculiar to such systems and 2) as an inseparable part of Lithuanian economy, its servicing infrastructure sub-system, creating by its functioning and development the conditions to expand other sectors of economy.

6. The employment of modern computer facilities. Modern computers allow sufficient arrangement of the dialogue "expert - modelling", to make multivariate calculations changing initial information, thus enabling to find a solution corresponding an expert's prediction or allowing him to make a reasonable correction. Meantime expert's participation in the dialogue (as that of a subjective factor) does not absolutely undermine the meaning of applied economical-mathematical models. Furthermore, a qualified expert may identify efficient directions how to alter parameters and structures of a complex system, check the expediency of these alterations, find weak points and formulate reasonable proposals for rational their elimination. Thus the synthesis of "subjective" and "objective" expert's knowledge may be achieved and intuition, his engineering state of art and formal methods of analysis. All this was actually performed with the help of expatriate experts for the elaboration of the "Lithuanian national transport system development programme".

Methods of systemic analysis enable to identify more reasonably the strategy of transport system development and technical policy in this field of economy, to select more efficient trends of transport technologies, most important parameters of transport development and characteristics: growth rates of transportation capacities, investments, the level of reserve in transport system, etc.

\section{Models of Transport Modes Interface}

Analysing the foreign literature of the last 10 years one may notice that the principal theoretical research of a transport system concerns the interface of different transport modes, for instance, the railway and road transport, the railway and maritime transport, etc.; it means - the combined freight transport.

The attention of specialists forecasting and elaborating different projects in this field is mainly focused on the systemic aspect of efficient use of different transport modes and most economical transport systems. Along the opportunities of employing new types of transport equipment were investigated as well as various modem means in the interface of transport systems analysed including buildings and structures necessary for handling and warehousing related to transportation process and reloading of freight from one type of transport to the other one.

The Lithuanian transport system development strategy foresees the investigation of the interface between all transport modes, namely: road, railway, maritime, inland waterways, pipeline and partially air transport (freight transportation by air transport is of the least proportions if compared with other transport modes). For this reason the whole number of models was adapted and principally changed (criteria of socialist commanding economy were not adequate). With the help of the above models calculations and investigations were carried out for the elaboration of the Lithuanian national transport development programme enabling the specification of its provisions at the present time as well as in the future.

For transport system modelling, for the assessment of costs for its efficient operability maintenance, a dynamic model was elaborated serving for the development of general transport system capacities. In the model there are the following denotements:

$G(t)$ - general costs for the period $t$;

$V(t)$ - transportation capacity of a transport system for the period $t$;

$m$ - comparative costs of the transportation capacity unit, the costs being necessary for uninterrupted functioning of transport system;

$f$ - comparable costs of a transportation capacity unit, necessary for renewal (replacement of present ones) of transport means;

$r$ - transportation capacity unit comparative costs necessary for the increase of transportation capacities;

$k$-coefficient of proportionality;

$K_{1}, K_{2}$ - constants of integration.

Transport system functioning general costs related 
to transportation capacity are:

$$
\begin{gathered}
G(t)=k V(t), \\
\text { here } \quad k V(t)=c V(t)+r V^{\prime}(t) \\
c=m+f .
\end{gathered}
$$

Having solved the differential equation in the $V(t)$ attitude we will obtain:

$$
V(t)=K_{1} 1 \frac{k-c}{r} t+K_{2}
$$

The latter model may be generalised by three cases of the assessment of costs necessary for the maintenance of transport system working ability.

I case. First generalisation - presenting general costs in the form of the linear equation:

$$
K_{1}(t)=a_{i}+b_{i} t, i=1, \ldots,(l) N,
$$

here $a$ - initial costs; $c_{i}$-coefficient of cost increase for a time unit. Coefficients $K_{i}(t)$ may be interpreted as linear functions for the maintenance of system work ability.

The function of general costs is put down as follows:

$$
G(t)=\sum_{i=l}^{N} K_{i}(t), i=1, \ldots,(l) N
$$

When $G(t)=a=$ const, we shall obtain:

$$
V^{\prime}(t)=\frac{a}{r}-\frac{c}{r} V(t)
$$

From this

$$
\begin{aligned}
& V(t)=\frac{a}{c}\left(l-c^{-\frac{c}{r} t}\right)+e^{-\frac{c}{r}-t}, \\
& c_{1}=\frac{a}{c}-K .
\end{aligned}
$$

In this expression the first member assesses the influence of external factors on the operation of a transport system (capital input into the development of the system main funds, etc.).

The second member characterises the influence of factors (aging of main transport means, structure of the main transport means fleet, etc.).

If $i=3$, we shall obtain:

$$
V(t)=i^{-\frac{c}{r} t}[c+A(t)]
$$

here $A(t)=\frac{K_{1}(t)+K_{2}(t)+K_{3}(t)}{r} \cdot e^{\frac{c}{r} t} d t$.

II case. The second generalisation - the coefficients of comparative costs which are divided into separate components for the sake of assessments of separate factors.

In this case the model of general costs is put down as follows:

$$
G(t)=\sum_{i}^{N} c_{i} V(t)+\sum_{i}^{M} r_{j} V^{\prime}(t), i=1(i) N, j=1(l) N .
$$

Computer technologies enable to analyse the real levels of specification during the processing of different forecasted data.

III case. The third generalisation - the assessment of the reversible impact of transportation capacities on the general costs.

Transportation capacities expansion enhances the realisation of transport services, as well as preconditions the growth of costs for system functioning, its work ability maintenance and modernisation.

Therefore the function of general costs will be this:

$$
G(t)=\sum_{i=1}^{S} g_{i} V(t), i=1(l) S .
$$

Coefficients $g_{i}$ may be analysed as coefficients of proportionality between the costs of transportation capacity and separate elements of the system. In turn, these coefficients may act as transportation capacity functions:

$$
g_{i}=u_{i}+z_{i} V(t), i=1(l) S,
$$

here $u_{i}$ - initial costs for maintenance of system work ability; $z_{i}$-coefficient of cost increase for a time unit.

For the assessment of efficiency of separate transport modes and for their mutual comparison it is necessary to have a system of indices characterizing the operation of a transport system, the coefficients of value of these indices and the rules of their aggregation into a uniform quantity.

From the whole set of indices the principal ones are selected characterising the efficiency of transport system operation:

- cost of transportation;

- time consumption for transportation (in monetary expression);

- time consumption for waiting (in monetary expression);

- cost of transportation unit.

The operation efficiency of a transport mode is assessed as follows:

$$
I_{E M}=\left(\alpha d_{1}\right)+\left(\beta d_{2}\right)+\left(\gamma d_{3}\right)+\left(\delta d_{4}\right),
$$

or, if detailed more specifically according to the transport modes, the route and freight types:

$$
\begin{aligned}
& I_{E M_{i, r, c}}=\left(\alpha_{i, r} c_{i} d_{1, i, r, c}\right)+\left(\beta_{i, r, c} d_{2, i, r, c}\right)+ \\
& \left(y_{i, r, c} d_{3, i, r, i}\right)+\left(\delta_{i, r, c} d_{4, i, r, c}\right),
\end{aligned}
$$

here $i$-index of transport mode; $r$-index of route; $c$ freight type index; $\alpha$-freight category; $d_{1}-$ transportation cost; $\beta$ - time consumption, $\mathrm{h} ; d_{2}-$ recalculation of freight unit transportation time consumption (into the cost in monetary expression); $y$ waiting time, $\mathrm{h} ; d_{3}-$ recalculation of freight unit waiting time into the cost (in 
monetary expression); $\delta$ - probability of freight nondelivery, its loss or damage; $d_{4}$ - cost of freight unit.

For the assessment of transport system operation efficiency in general it is necessary to sum up the meanings of separate transport modes obtained.

$$
I_{E M}=M_{1} I_{E M_{1}}+M_{2} I_{E M_{2}}+\ldots+M_{i} I_{E M_{i}} \rightarrow \min
$$

here $M_{i}=\sum_{g=1}^{G} m_{i}, c ; m_{i, c}-c(c=1, \ldots, C)$ type freight amount transported by the $i$-th transport mode; $M_{i}-$ general amount of freight transported by the $i$-th transport mode.

Having solved (13)-(15) it is possible to identify an efficient combination of transport modes. Whereas the assessment of transport modes is exercised as that of the whole transport network in general, the applied data is very aggregated. In transportation planning such an aggregation level is not always necessary. Usually it suffices to select the most efficient transport modes in one corridor existing in transport system. In such a case the problem is being solved:

$$
I_{E M}=M_{12}+M_{2} I_{E M_{22}}+\ldots+M_{1} I_{E M_{1}} \rightarrow \min ,
$$

when

$$
M_{i, r}=\sum_{g=1}^{G} m_{i, r, c},
$$

here $M_{i, r}$ - general amount of transportation performed by the $i$-th transport mode in the corridor $r ; M_{i, r, c}-c$ mode freight transportation performed by the $i$-th transport mode, in the corridor $r$.

Whereas all the indices are expressed by the cost, the application of models does not cause difficulties. Models may be used for the solution of complex problems, for instance, for the assessment of efficiency influence of separate transport means functioning.

\section{Determined Models of the Development of Trunk Transport Network}

Here we shall present a scalar optimisation problem of such formulation. Let us presume that we know the following:

a) the configuration of existing transport network and its possible topological changes during the analysed period;

b) different points of freight shipment and delivery, freight volumes (or an adequate correspondence matrix), also routes and quantities of passenger flows of different traffic categories;

c) the status of all elements of the network at the moment of planning, possible stages of their reconstruction (or new construction), as well as all necessary operational, technical and economic; characteristics enabling to identify every analysed element of the network (re- garding the amount and structure of transport operation performed by it) the identification of operational costs and capital input necessary for the development of the element to one or other level of capacity;

d) all additional restrictions the budgetary resources.

Having assessed the resource restrictions it is necessary to identify the means of development of the existing network and the construction of the new indispensable transport objects (as well as the corresponding terms of performing these means and carrying out new constructions), the presence of which will provide the least general costs for the change of network at the certain period and in that network all volumes of goods and passengers could be transported in the required terms and quality.

For the modelling of this issue different methods may be employed corresponding the different mathematical structure of economic-mathematical models. Further we shall analyse the ones that are the most common and applicable for the substantiation of the Lithuanian national transport system development programme. ables

\subsection{Static Model With Discrete-Continuous Vari-}

If we dissociate ourselves from the structure of transportation volumes and the changes of direction in time, then the model of a problem may be presented as follows:

$$
\min _{X, \eta} F(X, \eta)=\min _{X, \eta} \sum_{u} \sum_{k} f_{u k}\left(X, X_{\Pi}\right) \eta_{u k}
$$

When there are restrictions:

$$
\begin{aligned}
& S_{p} X=b \text {; } \\
& X \geq 0 \text { ； } \\
& \eta_{u k}=\left\{\frac{0}{1} \forall u, k ;\right. \\
& \sum_{k} \eta_{u k} \leq 1 \quad \forall u \text {; } \\
& X_{u}\left(1-\sum_{k} \eta_{u k}\right)=0 \forall u ; \\
& \sum_{u, k} \sum_{j u k} \eta_{u k} \leq R_{j} \quad \forall j,
\end{aligned}
$$

here $f_{u k}$ - function of brought out costs in the network element $u$, being $k$ level of its development (taking into account the costs for development); $X$ - initial vector of network loading by flows of all freight types; $X_{\Pi^{-}}$ defined vector of network loading with passenger transport; $b$ - vector of shipment-delivery amounts in the network peaks; $X_{u}$-loading vector of the network element loading $u ; \eta_{u k}$-identificator showing the network element $u$ status $k$ (if $\eta_{u k}=1$, then it exists, if $\eta_{u k}=0$-it does not exist); $\eta=\left\{\eta_{u k}\right\}$-the sought vector of the status of technical furnishing of network elements; 
$r_{j u k}$ - use of the resource $j$ in the network element $u$, seeking to lead it from the initial status into the $k$ status; $R_{j}$ - general permissible consumption quantity of the resource $j ; S_{p}-$ generalised incidents matrix corresponding the flows of transportation of non-uniform freights: $S_{p}=\left|i S_{i j l}\right|$, whereas $l=1,2, \ldots, p$;

$$
S_{i j l}=\left\{\begin{aligned}
&+1, \text { if the radius of the network goes out from the } \\
& \text { node } i \text { and it is possible to perform } \\
& \text { by it the transportation of } l \text { type freight } \\
& \text { (total number of freight types - } p \text { ); } \\
&-1, \text { if the radius } j \text { goes enters into the node } i \text { and it } \\
& \text { is possible to bring by it the freight } l ; \\
& 0 \text { - other cases. }
\end{aligned}\right.
$$

Thus, according to the purpose function (18) general brought out costs are minimised when there are restrictions:

a) restrictions of a technological type (18) and (19) - condition that all transportations should be performed;

b) for reconstruction: restrictions (21)-(22) - every element may be only in one status, and if the element is not created, then the work performed by it equals to zero - restriction (19)-(24);

c) resource restriction (24) - every resource type may be used within the limits of defined amounts.

Solving the problem (18)-(24) the list of means of the optimal development of the network is obtained however, their implementation terms are not identified as well as their stages, i.e. a successive change in the time of the level of elements of technical status.

\subsection{Dymamic Model With Discreet-Continuous Variables}

Formulating the dynamic problem we shall consider the identified freight shipping and delivery time changes of (vector $b_{t}$ ), the intensity of network loading in passenger flows $X_{n}^{t}$, the influence of scientific and technical advance, as the well as impact of other brought out costs for the function $f_{u k}^{t}\left(X^{t}, X_{n}^{t}\right)$.

We shall also consider that:

a) possible technical supply states of each element of a network are partially ordered (for instance, in capacity growth) and this order is realised in time, i.e. passing to the lower levels is not foreseen;

b) the cost of "situation rescue" in changing the state of elements is small, i.e. the investment necessary for passing of element from one state to another one directly or through oîher intermediate hierarchic links of a state does not change much;

c) the amount of operational costs of transportation performed by any element of the network is identified for each entire year according to the technical supply and loading of each separate element of a network.
Then the dynamic model is natural generalisation of the model (18)-(24) and will be:

$$
\begin{aligned}
& \min _{X^{t}, \eta^{\prime}} F\left(T, X^{t}, \eta^{\prime}\right)= \\
& \min _{X^{t}, \eta^{\prime}} \sum_{t=1}^{t=T} \sum_{u, k} f_{u k}^{t}\left(X^{t}, X_{n}^{t}, \eta^{t} \bigcap_{u k}^{t}(1+E)^{-t} .\right.
\end{aligned}
$$

When there are restrictions:

$$
\begin{aligned}
& S_{p}^{t} X^{t}=b^{t} ; \\
& X^{t} \geq 0 ; \\
& \eta_{u k}^{t}=\left\{\frac{1}{0} \forall u, k, t ;\right. \\
& \sum_{u k}^{t} \leq 1 \forall u, t ; \\
& X_{u}^{t}\left(1-\sum_{k} \eta_{u k}^{t}\right)=0 \quad \forall u, t ; \\
& \sum_{t=\theta_{1}}^{t} \sum_{u, k} r_{j u k}^{t} \eta_{u k}^{t} \leq R_{j}^{\theta_{1}, \theta_{2}} \forall j ; \\
& \left(\sum_{k}^{\left.\sum k \eta_{u k}^{t}-\sum_{k} k \eta_{u k}^{\tau}\right)}\right\}_{t-\tau) \geq 0 \quad \forall k, u, t, \tau,}
\end{aligned}
$$

here $t, \tau$ - index of the current year; $T$ - length of the accounted period; $R_{j}^{\theta_{1}, \theta_{2}}$ - restrictions to the resources $j$ for the general period of use since the year $\theta_{1}$ until the year $\theta_{2}$ inclusively (usually $\theta_{1}$ - the year of the beginning of planning, and $\theta_{2}$ - the year of the finish of planning), if the restriction (31) is defined for a certain year separately, then $\theta_{1}=\theta_{2}=t$. While defining in the inequality (31) existing meanings $r_{j u k}^{t}$ (resource amount is necessary to lead the element $u$ in the year $t$ from initial state into the state $k$ ) then as an initial state the state $(t-1)$ in the end of the year is taken.

The essence of the purpose function (25) and restrictions (26)-(31) are analogous (only analysed in dynamics) to the corresponding relations (18) and (19)-(24) in static model. The condition (32) defines the order of the possible change of a technical state of elements of the system.

Solving the problem (25)-(32) it is possible to define in what year which elements of which state should be reconstructed (or built). In this case the time is assumed as the discrete one, i.e. the pace of change of amounts $t, \tau, \theta_{1}, \theta_{2}$ equals to one year.

\section{3. Dynamic Model With Continuous Variables}

Principally it is possible to eliminate discrete variables of the dynamic model and to accept the time as un- 
interrupted. The corresponding model is formulated as follows (restrictions of resources eliminated):

Let us presume that in the peaks of the graph describing the transport network the time functions will be defined so that at any moment of time the balance of production and consumption of each freight will be ensured.

Panes of the graph correspond to certain network stretches, each of them being one of several technical states numerated by the indices $k=0,1, \ldots$ In the reconstruction of network stretches the meanings $k$ for each pane $u$ of graph may change along with the time, as the state of network stretch corresponding to the link $u$, is described by the function $k_{u}(t)$ having the meanings of whole numbers.

We shall denote the fracture points of this function by $\Theta_{u z}$, here $z=1, \ldots, n_{u}$ and we shall consider it uninterrupted from the left. For simplification we shall assume that during the moments used investments of the amount $K_{u}\left(k_{u}\left(\Theta_{u z}\right), k_{u}\left(\Theta_{u z}+0\right)\right.$, here $K_{u}\left(k, k^{\prime}\right)$ - the defined function of arguments of two whole numbers for all possible pairs.

Freight transportation from the points of production to the points of consuming in the course of time forms changing flows and the graph panes which instant intensity at the moment $t$ further is marked as $X(t)=\left\{X_{u}(t)\right\}$, here $X_{u}(t)$ - the vector of transportation of freight of all types in both directions in the chain $u$. For the transportation in the stretch current costs are required, which depend upon the technical state of the stretch and on the intensity of transportation in it. We shall indicate the amount of these costs by $f_{u}\left(k_{u}, X\right)$.

The issue of transport network development optimisation at a planned period $(0, T)$ in the simplest case has the following shape:

$$
\begin{aligned}
& \min _{X, \theta}\left\{\sum_{u} \sum_{z=1}^{z=n_{u}} K_{u}\left(k_{u}\left(\theta_{u z}\right), k_{u}\left(\theta_{u z}+0\right)\right)(1+E)^{-\theta_{u k}}+\right. \\
& \left.\int_{0}^{T} f_{u}\left(k_{u}(t), X(t)\right)(1+E)^{-t} d t\right\}
\end{aligned}
$$

on condition

$$
\begin{aligned}
& S_{p}(t) X(t)=b(t) \forall t \in[0, T] ; \\
& X(t) \geq 0,
\end{aligned}
$$

here $X(t)$ is the sought transportation intensity vector function; $b(t)$ is in the peaks of the graph defined vector function of intensity of all freight production-consumption.

As it is visible from the model all sought variable quantities $X$ and $\theta$ change uninterruptedly. However, actually it is only another form of denoting relations presented in a discrete form in the model (25)-(32).

The methods of analysis of models (25)-(32) and (33)-(35) are very similar: they base on the procedure of two stages when at the beginning the state of the network is fixed and the flows are optimised and afterwards flows are fixed and the state is optimised.

\section{4. Modelling of the Trunk Transport Network} Development under Conditions of Indeterminacy

Objectively, indeterminacy is characteristic to complex transport systems. It most often manifests itself through the imperfection (and uncertainty) of the initial information of technical-economic characteristics of systems and their operation as well as development conditions. For instance, in variant calculations of transport network development used massive norms for definition of operational costs and investment have a bias of 15 $20 \%$. The forecasts of transportation amounts and structure have more significant biases from the reality.

Inadequacy of economic-mathematical models to the real transportation process adds more (modelling) indeterminacy, the totality of all types of which exercises an essential impact on optimisation results.

Planning the development of complex systems under the conditions of indeterminacy one of the most essential elements is the compression of an adequate indeterminacy zone with the help of series of optimisation calculations performed for various conditions of system functioning and with various criteria. In general this methodology has the following stages:

1) the analysis of imperfection of the initial information, the identification by the most essential correlations of its component separate parameters and their groups;

2) the formation of the most proper combinations of meanings of the initial information (aprioric and by experimenting with a model, for instance, the application of the Monte Karlo methods);

3) the election of the type of a determined calculation model, of collections of restrictions of the most important optimisation criteria, etc.;

4) multiple calculations performed with the aim to define optimal plans corresponding to the type of each model;

5) the compression (with the help of formal and informal methods) of thus obtained set of optimal plans into the set of optimal strategies, i.e. the identification of indeterminacy zone;

6) the assessment of efficiency of different strategies of indeterminacy zone (making assessment of adequate costs for adaptation by the realisation of certain apriori not known meanings of initials information) and the selection of the final decision made, as a rule, in the expertive manner.

The character of multileveled models of transport system development decides upon certain specifics in the performance of optimisation exercised under conditions of indeterminacy. It should be stressed that the calculation in higher hierarchic levels is expedient for the analysis not of all, but only of the functioning regimes of elements of possibly efficient lower hierarchic level. There- 
fore, it is expedient to define certain equivalent aggregated characteristics of elements. The characteristics later on are used in further calculations. For these calculations the following features are characteristic:

- massive nature and certain unitypicality - usually sought collection of parameters ensuring the performance of necessary transportation in set terms with minimal general brought out costs;

- decentralised nature of calculations and independence of interplay results;

- relatively not big tasks, namely, the usage of common funded resources according to the task formulation usually is not limited (for instance, that of investments, labour resources, fuel and electric power consumption).

Traditional scheme, assessing the indeterminability in task solving, looks as follows:

1) an expert analysis of initial information is performed and its normative meaning is defined according to the most important positions (for instance, in comparison of the traction efficiency of electrically driven vehicle with a diesel traction locomotive; it is related to the costs of diesel fuel and electric energy);

2) basing on the normative initial information determinated optimisation calculation is performed aiming to find out the best variant and the assessment of its characteristics;

3) variation of the initial data is performed with the aim to assess the found out best variant;

4) for decision-making experts use the obtained results on the comparative effectiveness together with additional restrictions.

It is purposeful to shift from a traditional scheme of indeterminacy to the direct finding of averages of the expected losses; this may be reached after introduction of probability assessment and after the admitting of general brought out costs as criteria of optimality or minimum of mathematical probability of economic risk.

It seems more purposeful to shift to mathematical probability, because here massive calculations are dealt with (though with individual realisation) according to relatively insignificant tasks solved independently. Whereas indeterminacy occurs not because of energetic actions of a opponent, but only because of an unknown initial state. Thus, it is possible to state that in the whole system of these calculations there are conditions close to those when the law of complex numbers is in effect and the criterion of medium risk here is much better than the minimaxic criterion.

Probability assessment may be performed by the manner of experts' reasoning or formally, for instance, on the basis of the principle of maximum entropy.

After the introduction of probability assessment optimal functioning regimes of the lower level transport objects and sequence may be identified, forming adequate characteristics for solving of higher hierarchic level tasks (mathematical probability $f_{u k}$ of functions of brought out costs, etc.).
Then, having assessed the factor of indeterminability, the static model (18)-(24) may be denoted as follows:

$$
\begin{aligned}
& \text { opt } F(X(\omega), \eta(\omega))= \\
& X, \eta \\
& \operatorname{opt} \sum_{u} \sum_{k}\left(\tilde{f}_{u k}\left(X(\omega), X_{\Pi} \eta_{u k}(\omega)\right),\right.
\end{aligned}
$$

when restricted:

$$
\begin{aligned}
& S_{p} X(\omega)=b(\omega) ; \\
& X(\omega) \geq 0 ; \\
& \eta_{u k}(\omega)=\left\{\frac{0}{1} \forall u, k, \omega ;\right. \\
& \sum_{k} \eta_{u k}(\omega) \leq 1 \forall u, \omega ; \\
& \operatorname{Pr}\left\{\sum_{k} \sum_{u} r j_{u k}(\omega) \eta_{u k}(\omega) \leq \tilde{R}_{j}\right\} \geq 1-\varepsilon,
\end{aligned}
$$

here opt - the symbol of optimisation procedure; $\omega-$ concrete possible realisation from $\Omega$ given set of conditions of indeterminacy; $\mathrm{Pr}$ - probability; $\varepsilon$ - limit of significance; $X(\omega), \eta(\omega)$ - sought vectors of network loading and the state of its elements.

The single stage task (36)-(41) allows different modifications of formulation, interpretations and procedures of search for optimal development strategy (correspondingly according to the determinate and probability plans).

Generalised formulations are good for multistage case when the notion of the plan may be wholly transformed into the plan-instruction in the managed random process.

It should be noticed that using probability models the calculations according to them may give essentially different results than the calculations according to the determined models following the mathematical probabilities of random parameters. There are many reasons for this, the most important of them are non-linearity of dependencies and correlation between the parameters. This is particularly important to assess in rationing the transportation process start-finish duration, traffic and other operations.

\section{The Model of the Development of Private Freight Road Transport}

In market economy private road transport has to develop constantly and to satisfy the public demands. Development trends have to be foreseen forecasting freight flows. In the course of the integration of private freight road transport into the general transport system the important issue of forecasting of transport amounts is the 
forecasting of these amounts to other transport modes as well (railway, water and air transport), whereas the freight road transport, except its direct operation, also serves the railway, water and air transport.

For the models of private freight road transport development non-stationary processes are characteristic. The processes of private freight transport development under competition may be analysed as the process of proliferation and fall which in literature is well analysed and described [8].

One of the most realistic and general tasks arising in the course of analysis of private freight transport development consists of the elaboration of the characteristics of use of resources of this transport. From the point of view of the process of proliferation and fall such a task corresponds to the calculation of the probability that the time needed for the process to reach a required state $n$ (if the process starts from the state $i$ ) exceeds the defined time $T$. According to the Chabischev's inequality:

$$
P_{i}\left\{\tau_{n} \geq T\right\}=P_{i}\left\{\tau_{n}-M_{i} \tau_{n}>T-M_{i} \tau_{n}\right\} \leq \frac{D_{i} \tau_{n}}{\left(T-M_{i} \tau_{n}\right)^{2}},
$$

here $M_{i} \tau_{n}$-mathematical probability of the time through which there is reached the state $n$, starting from the state $i$, and $D_{i} \tau_{n}=M_{i} \tau_{n}^{l}-\left(M_{i} \tau_{n}\right)^{2}-$ the dispersion of this time.

For $M_{i} \tau_{n}$ and $D_{i} \tau_{n}$ calculation we shall deduce the equation satisfying the function $m(x)=M_{x} \tau_{n}$ and $d(x)=M_{x} \tau_{n}^{2}$. Through the time $d t$ in the trajectory of the process being in the point $x$, there may emerge one of three situations (if we do not count the situations the probability of which is $O(d t)$.

Situation $A_{1}$. Process remains not changed with the probability $1-\left(\lambda_{x}+\mu_{x}\right) d t+O(d t)$.

Situation $\boldsymbol{A}_{2}$. Process passes into a neighbouring state with the probability $\lambda_{x} d t+O(d t)$.

Situation $A_{3}$. Process passes into the former neighbouring state with the probability $M_{x} d t+O(d t)$.

According to the formula of total mathematical probability we shall find:

$$
\begin{aligned}
& m_{i}=P_{i}\left(A_{1}\right) M_{i}\left(\tau_{n} / A_{i}\right)+P_{i}\left(A_{2}\right) M_{i}\left(\tau_{n} / A_{2}\right)+ \\
& P_{i}\left(A_{3}\right) M_{i}\left(\tau_{n} / A_{3}\right)+O(d t)= \\
& =\left(1-\left(\lambda_{i}-M_{i}\right) d t+O(d t) M_{i}\left(\tau_{n}+d t\right)+\right. \\
& \lambda_{i} d t M_{i+1}\left(\tau_{n}+d t\right)+\mu_{i} d t M_{i-1}\left(\tau_{n}+d t\right) .
\end{aligned}
$$

If we do not assess the succession by the amount $O(d t)$ and will perform abbreviation, we shall obtain the equation system:

$$
\lambda_{i} m_{i+1}+M_{i} m_{i 1}-\left(\lambda_{i}+\mu_{i}\right) m_{i}=-1
$$

here

$I=1,2, \ldots, n-1, m n=0$.

Analogically the equation system is obtained

$$
\lambda_{i_{i d_{i+1}}}+M_{i d_{i-1}}-\left(\lambda_{i}+\mu_{i}\right) d_{i}=-2 m_{i}
$$

here $i=1,2, \ldots, n-1, d n=0$. When $i=0$ condition $\mu_{0}=0$, the above lines acquire the shape:

$$
\lambda_{0}\left(m_{1}-m_{0}\right)=-1, \lambda_{0}\left(d_{1}-d_{0}\right)-2 m_{0} .
$$

Whereas the equations (42) and (43) are uniform, firstly we shall solve the equation by the following general shape:

$$
\lambda_{i w_{i+1}}+\mu_{w_{i-1}}-\left(\lambda_{i}+\mu_{i}\right) \omega_{i}=-f_{i}
$$

here

$i=0,1, \ldots, n-1, \omega_{n}=0$, not specifying the shape of the member $f_{i}$. Having introduced the note:

$$
U_{i}=\omega_{i}-\omega_{r+1}
$$

From (44), when $i=0$, we shall obtain $\lambda_{0} U_{0}=f_{0}$, and when $i>0 M_{i} U_{i-1}-\lambda_{i} U_{i}=-f_{i}$, from which $U_{i}=\left(\mu_{i} U_{i-1}+f_{i}\right) / \lambda_{i}$. Thus

$$
\begin{aligned}
& U_{0}=f_{0} / \lambda_{0}, \\
& U_{1}=\mu_{1} / \lambda_{1} \lambda_{0}+f_{1} \lambda_{1}, \\
& U_{2}=f_{0} M_{1} M_{2} / \lambda_{0} \lambda_{1} \lambda_{2}+f_{1} M_{2} / \lambda_{1} \lambda_{2}+f_{2} / \lambda_{2}, \\
& U_{1}=P_{i} \sum_{j=0}^{i} \frac{f_{i}}{\lambda_{j} P_{j}},
\end{aligned}
$$

here $P_{0}=1, P_{i}=\mu_{1}, \ldots, M_{i} / \lambda_{1}, \ldots, \lambda_{I}$.

Having put the equations together (45), starting with the meaning $U_{i}\left(\omega_{i}\right)$ until the meaning $U_{n-1}\left(\omega_{n-1}\right)$ and having assessed that $\omega_{n}=0$, we shall obtain:

$$
\begin{aligned}
& \omega_{i}-\omega_{n}=\sum_{j=1}^{n-1} U_{j}=\sum_{j=1}^{n-1} P_{j} \sum_{k=0}^{j} \frac{f_{k}}{\lambda_{k} P_{k}}, \\
& \omega_{i}=\sum_{j=i}^{n-1} P_{j} \sum_{k=0}^{j} \frac{f_{k}}{\lambda_{k} P_{k}} .
\end{aligned}
$$

With the help of the formula (47) it is possible to find the meanings $m_{i}$, and $d_{i}$. Having inscribed $f_{i}=1$, we find:

$$
m_{i}=\sum_{j=1}^{n-1} P_{j} \sum_{k=0}^{j} \frac{1}{\lambda_{k} P_{k}}
$$

and having inscribed $f_{i}=2 m_{i}$, we find:

$$
\begin{aligned}
& d_{i}=2 \sum_{j=1}^{n-1} P_{j} \sum_{k=0}^{j} \frac{m_{k}}{\lambda_{k} P_{k}}= \\
& 2 \sum_{j=1}^{n-1} P_{j} \sum_{k_{0}}^{j} \frac{1}{\lambda_{k} P_{k}} \sum_{l=k}^{n-1} P_{l} \sum_{m=0}^{l} \frac{1}{\lambda_{m} P_{m}} .
\end{aligned}
$$

With the help of the formulae (48) and (49) it is possible to calculate $D_{i} \tau_{n}=d_{i}-m_{i}^{2}$ and later on, having used 
the Chabyschev's inequality it is possible to calculate the probability of preservation of private freight transport work ability (without reloading) for the set time.

Further to the analysed task (general characteristics of the developing private freight transport), the characteristics of resource use per one time unit, for instance, per year (increase of loading) should be known.

For the analysis of the above characteristics [3] the so called proliferation and fall process "with migration" characterised by the shifting probabilities is introduced.

$$
\lambda_{i}=i \lambda+\beta, M_{i}=i M,
$$

Here the constanta $\beta$ is introduced with the aim of avoiding the interruption of the process in case it reaches the zero status at some time. In a certain case, when $\beta=0$, this process converges into the Candel's process (i.e. into the process when the intensities of the both shiftings are proportional to the number order of the status) [8].

Let us denote $b(t)=M_{b n}$ - the medium proliferation number per time $(0, t), d(t)=M_{d n}$ - the medium number of the fall per time $(0, t)$. Then the medium amount of increase will be $m(t)=i+b(t)-d(t)$, here $d(t)$ - the status of the process at the time moment 0 .

Having expressed by $t$ year it is possible to identify annual increase:

$$
q(t)=(m(t+1)-m(t)) / m(t) .
$$

Having used [8] it is possible to find the annual increase:

$$
q(t)= \begin{cases}\frac{[i+\beta / \Delta] e^{\Delta t}\left(e^{\Delta}-1\right)}{[i+\beta / \Delta] e^{\Delta t}-\beta / \Delta}, & \text { when } \lambda \neq \mu \\ \beta /(i+\beta t), & \text { when } \lambda=\mu,\end{cases}
$$

where the admitted noting is $\Delta=\lambda-\mu$. It is evident that for the selection of the coefficient it is possible to define the increase ratio. In the course of time the medium increase may be defined:

$$
\bar{q}=\left\{\begin{array}{l}
0, \text { when } \lambda<\mu, \beta \neq 0, \\
0, \text { when } \lambda=\mu, \\
e^{\Delta}-1 \text { in other cases. }
\end{array}\right.
$$

Having got the statistical data and the algorithm of the task solution, it is possible to model the demand for suitable transport means.

\section{Conclusions}

1. The Lithuanian national transport development programme outlines only the qualitative characteristics of the development, therefore for the implementation of the programme, i.e. for the identification of the quantitative characteristics systemic investigations based on the statistical data are necessary. They could make separate research themes.
2. Wishing to adjust to the demands of freight consignors, i.e. seeking to facilitate their choice of a carrier by optimising tariffs according to transport modes or combined transportation, it is necessary:

- to arrange reliable interaction of separate transport modes for the improvement of a technological process of transportation - by the implementation of modern terminals, handling points, etc.;

- to improve throughput capacity of separate transport modes and better use of transportation capacities

3. For the assessment of the full-scale amount of transport system operation the general circulation indicator $P=\sum_{k} P^{k}=\sum_{k, r} P_{r}^{k}$ and general transportation volume $Q=\sum_{r} Q^{r} \quad k$ should be used, since it may be larger than the initial volume of freight consignment.

4. For the assessment of transport development efficiency by costs and results the following principle has to be observed: the costs spent for transport development have to be no less than the efficiency which would be received using the funds in some other branch of national economy.

5. Statistical accountancy system of the Lithuanian transport activities should be reorganised essentially. One of the main features of this system should be the ability to assess the losses of the entire economy caused by the low standards of transport development and its nonqualitative functioning. For this reason calculation models ought to be created and selective research ought to be performed.

6. Having performed the research according to the suggested methods of systemic analysis and having solved certain tasks of transport activities modelling, quite reliable priorities were defined, i.e. transport infrastructure objects of Lithuanian economy which should be developed.: Lithuanian railways, Klaipeda State Sea Port Authority, State Flight Management Authority, Vilnius Air Port, Kaunas Air Port, VIA BALTICA highway.

7. Having performed the analysis of the Lithuanian transport development strategy from the example of international transportation performed by the road transport, it may be stated that the selected strategy of privatisation of this transport is proper and the results correspond to the situation existing in the Western Europe and Scandinavian countries.

8. An essentially new private freight road transport development model has been created which in future, after having been mastered and correspondingly developed, will enable forecasting of the demand for transport rolling stock of various modes.

9. The interaction of different transport modes, their interests, aims and criteria of assessment of the mechanism of functioning, decide upon the occurrence of systemic effects, i.e. parameters which cannot be separated and assessed according to the separate characteristics of transport modes and may be defined only in their interaction. 
The assessment of systemic effects is very important for the analysis of complex transport because of the fact that the orientation towards the local effect of different transport modes leads to the solutions which are not always useful to the entire system. Therefore, a relevant mechanism for co-ordination of interests is necessary. The co-ordination of this mechanism has to guarant the expediency of solutions to users of the entire transport system. In such situation there may be certain cases when the efficiency of the entire system does not coincide with the sum of efficiencies of its separate elements.

10. The results obtained using the dynamic model for identification of general transport system transportation capacity development, as well as the forecasts of freight flows until the year 2020 in the West - East and South - North directions, convincingly demonstrate (the volumes of international freight transportation will increase by $110 \%$ ) the necessity to develop the multimodal transportation volumes of the Lithuanian transport system in the following priorities: 1) road transport - railway transport - maritime transport; 2) road transport - railway transport. Data - that of the basic year as well as that of the forecasted year, demonstrate that the West - East flows will be prevailing transit flows with Russia (per day, by the Klaipeda ferry about 150 road transport means will be transported related to Russia), Germany and the countries of Benelux. In the South - North directions the freight flows related to Finland, Sweden, Poland and Germany will prevail.

11. Decision-making in the transport system development should be guaranteed by the co-ordination of its interacting branches. However, the reiterative procedure of co-ordination of local and global optimums should be performed with the help of preparatory optimisation (assessment of resources) basing on the development schemes and operation regimes of all the elements of the system. In other words, optimal synthesis of transport system should foresee the development and optimal functioning of its separate elements.
12. The capacity of elements of a transport system as a rule has to bypass that quantity of it which is directly planned for this period basing on the transportation capacity forecasted for this time. The proportion of increase (reserve) is approximately defined by the following three main factors: discreet scale of capacities of transport objects, fluctuation of transportation demands within the time, available at that time incomplete information about them. Optimal quantity of the reserve has to be identified basing on all the losses caused by the minimum sum of failure to perform transportation or their not timely performance, i.e. when the demand for transportation exceeds the effective transport abilities and additional costs of making relevant reserve and functioning.

\section{References}

1. Southworth F.; Peterson E. Intermodal and international freight network modelling. In: Transportation research, Part C 8, 2000, p 147-166.

2. Zhan F.-B.; Noon Ch.-E. Shortest Path Algorithrns: An Evaluation using Real Road Networks. Transportation Science, Vol 32, No 1. February, 1998, p 65-73.

3. Mingozzi A.; Baldacci R.; Ball M. The Rollon-Rolloff Vehicle Routing Problem. Transportation Science, Vol 34, No 3, 2000, p 258-271.

4. Crainic T.; Florian M.; Leal J. A Model for the Strategic Planning of National Freight Transportation by Rail. Transportation Science, Vol 24, No 1, 1990, p 1-39.

5. Baublys A. Introduction to the theory of transport systems (Transporto sistemos jvadas). Vilnius: Technika, 1997 (in Lithuanian).

6. Baublys A. Strategic planning of freight tail transport. Transport Engineering (Transportas), Vol XIV, No 5, Vilnius: Technika, 1999, p 209-214.

7. Livshitz V. N. Systemic analysis of economic processes in transport. M.: Transport, 1986. $240 \mathrm{p}$ (in Russian).

8. Neiman V. I. Theoretical essentials of the uniform automated communications network. M.: Science, 1984. 367 p (in Russian). 